the high energy chemistry associated with turbulent flow of liquids. These chemical effects result from cavitation: the creation, growth and implosive collapse of bubbles in liquids ${ }^{2}$. The implosive collapse of bubbles can generate intense, but transient, local heating ${ }^{3}$ of the order of $5,000 \mathrm{~K}$. This is sufficient to induce the homolytic cleavage of $\mathrm{O}-\mathrm{H}$ bonds of water $^{4}$ and $\mathrm{C}-\mathrm{C}$ bonds of hydrocarbons ${ }^{5}$. Cavitation is usually associated with ultrasonic irradiation, but it also occurs during any turbulent flow. For example, Anbar demonstrated many years ago ${ }^{6}$ the existence of cavitation and associated high energy chemistry during turbulent mixing of water. The effects of cavitation and associated shock waves on biological systems can be dramatic, leading to cell rupture and death as the limiting case ${ }^{7}$.

We suggest that the degranulation observed by Benveniste and coworkers is an artefact of cell damage caused by reactions with small amounts of $\mathrm{OH} \cdot, \mathrm{H} \cdot$, $\mathrm{H}_{2} \mathrm{O}_{2}, \mathrm{HO}_{2}$, etc., produced by their use of vortex turbulence. Our hypothesis is easily tested: do basophils degranulate upon addition of water or buffered solutions previously subjected to vortex mixing, to high speed propeller cavitation (from a turbine homogenizer), or to high intensity ultrasound (from a cell disruptor)? The treated water should not contain any protein, which could serve to scavenge reactive species. Alternatively, do atmospheres of helium or carbon dioxide suppress the observed effects? These gases dramatically diminish the temperatures reached during cavitational collapse and suppress most chemical effects.

Kenneth S. Suslick School of Chemical Sciences,

University of Illinois,

Urbana,

Illinois 61821, USA

1. Davenas, E. et al. Nature 333, 816-818 (1988).

2. Suslick, K.S., ed. Ultrasound: Its Chemical, Physical, and Biological Effects (VCH. New York, 1988).

3. Suslick, K.S., Hammerton, D.A. \& Cline, R.E., Jr J. Am. chem. Soc. 108, 5641-5642 (1986)

4. Makino, K., Mossoba, M.M. \& Riesz, P. J. phys. Chem. 87, 1369-1377 (1983)

5. Suslick, K.S. \& Flint, E.B. Nature 330, 553-555 (1987).

6. Anbar, M. Science 161, 3848 (1966).

7. Williams, A.R. Ultrasound: Biological Effects and Potential Hazard (Academic, New York, 1983).

SIR-Davenas et al. ${ }^{1}$ observed repetitive waves of degranulation of human basophils, which had been exposed to increasing dilutions of anti-immunoglobulin $\mathrm{E}$ (anti-IgE) antibody. Degranulation was reported at the highest dilutions, even in the calculated absence of anti-IgE antibody. The hypothesis was suggested that the molecular organization of water might have been responsible for transmission of biological information. There may be a simpler explanation, more readily accommodated by the existing scientific paradigms.
Davenas et al. 'reported using Tyrode's solution to dilute antibody. As gleaned from their paper, this solution always appeared to contain heparin. Heparin has a number of interesting properties that are germane to what may be a novel role in mediating the activity of anti-IgE antibody. Heparin exists in a helical configuration, binding water molecules, as well as a number of monovalent and divalent cations (including $\mathrm{Na}$ and $\mathrm{K}$, which are present in Tyrode's solution) ${ }^{2}$. It also binds to various proteins and synthetic polypeptides $^{2.3}$. Like other glycosaminoglycans, heparin chains are further capable of interacting with one another, forming molecular aggregates ${ }^{3}$.

I propose that anti-IgE antibody (or any of the other immunological stimuli noted in the paper ${ }^{\mathrm{i}}$, that were responsible for basophil degranulation) might have acted as a template for heparin, thereby inducing a specific conformation of the heparin molecule. This molecular conformation would then be stabilized, perhaps by interacting with another heparin molecule. Upon dilution with heparin-containing Tyrode's solution, the stabilized heparin conformation, although lacking biological activity, would itself serve as a template, effecting a new heparin conformation which would mimic the threedimensional structure of the antigen-binding site of anti-IgE antibody (or other immunological stimulus). Presumably water molecules and perhaps the cations present in Tyrode's solution would stabilize this new, antigen-binding heparin conformation. Subsequent dilution with fresh heparin would, however, result in the antigen-binding conformation further acting as a template for formation of the biologically inactive conformation. Successive dilutions would generate the alternating heparin conformations.

Bionix Corporation,

J. LESLIE GLICK

10899 Deborah Drive,

Potomac,

Maryland 20854, USA

1. Davenas, E. et al. Nature 333, 816-818 (1988)

2. Chakrabarti, B. \& Park, J.W. Crit. Rev. Biochem. 8, 225$313(1980)$.

3. Lindahl, U. \& Höök, M. A. Rev. Biochem. 47, 385-417 (1978).

SIR - There may be a very simple explanation of Benveniste's conclusion (Nature 333, 816; 1988). Imagine there is some degranulating active molecule, not necessarily an anti-IgE, that binds to one component of Hepes-Tyrode's solution, for example heparin or EDTA; then there will not be a real dilution of the active compound.

Imagine that the binding is or is not reversible depending on the degree of "vigorous shaking", you get an explanation for "rhythmic fluctuation" in the activity (not so rhythmic in fact). This explanation is more comfortable than 'water memory' or throwing away the Law of Mass Action or Avogadro's number.

M.J. EsCRIBANO

CNRS Groupe de Laboratoires de

l'Institut de Recherches Scientifiques sur le Cancer,

7 Rue Guy-Môquet,

BP No. 8 ,

94802 Villejuif Cedex,

France

\section{Creationism and evolution}

SIR-Andrew P. Whipple (Nature 333, $492 ; 1988)$ tries to take an even-handed approach towards science and creationism, but in my opinion fails in this attempt. First, he fails to give weight to the coherence between theory and observation that evolutionary theory is alone able to offer. It is this observationally multicentred coherence that gives scientific 'belief' a privileged character, not shared by other types of belief. Furthermore, Whipple gives a truncated account of the "world views' in presence. He defines the naturalistic viewpoint as one of two basic positions, namely one that asserts that there is no reality beyond the physical and that denies the supernatural. This he opposes to the theistic viewpoint. In fact, there is a third position, not covered by your correspondent's analysis, which represents a different naturalistic view. This view also excludes the supernatural, but holds that the spiritual domain has a reality of its own as much as the world of phenomena does, a reality subject to the equivalent of natural laws, that is, engaged in obligatory, if unknown, relationships with the world of phenomena. These relationships need not be more arbitrary or inconstant than the relationships within the observable world itself and therefore are not in favour of a personalized and humanized god. The view I refer to holds that the mind, fully as real as, and distinct from, observable nature, is another side of nature. (By observable nature, I refer to the nature before us, not necessarily totally observable.) Our present ignorance of what this other side of nature is does not dispense us from acknowledging the existence of a philosophical position that is opposed by creationism, yet is not 'materialistic', but 'naturalistic' in a sense different from your correspondent's.

\section{Linus Pauling Institute}

EMILE ZUCKERKANDL

of Science and Medicine,

440 Page Mill Road,

Palo Alto,

California 94306, USA

Letters submitted for Correspondence

should be typed and double-spaced. 